\title{
Um modelo para monitoramento de sinais vitais do coração baseado em ciência da situação e computação ubíqua
}

\author{
Cristofe C. Lopes da Rocha ${ }^{1,2}$, Cristiano André da Costa ${ }^{1}$, Rodrigo da Rosa Righi ${ }^{1}$ \\ ${ }^{1}$ Programa de Pós-Graduação em Computação Aplicada - \\ Universidade do Vale do Rio Sinos (UNISINOS) \\ ${ }^{2}$ Departamento de Pesquisa e Pós-Graduação e Inovação - \\ Instituto Federal de Roraima (IFRR) \\ cristofedifrr.edu.br, \{cac,rrighi\}@unisinos.br
}

\begin{abstract}
Patients with heart failure and without daily monitoring may have heart vital signals committed, becoming inevitable their hospitalization. This recurrent scene decreases the patient's life quality, resulting in hospital readmissions generating costs to health of system. The use of ubiquitous care, employs sensors and wearables, can automate this process reducing the number of hospital admissions. In this context, we are proposing a model named Ubheart, which employs situation awareness to identify possible heart problems. As a scientific contribution the proposed model monitors the possible degradation of patient's heart vital signs, using the detection of situations of cardiac complications. To accomplish this, we have used health parameters established by the Brazilian Society of Cardiology. The Ubheart evaluation was conducted in two usage scenarios, showing satisfactory and encouraging results.
\end{abstract}

Resumo. Pacientes com insuficiência cardíaca e sem acompanhamento médico diário podem ter os sinais fisiológicos do coração comprometidos, causando graves problemas de saúde. Este cenário recorrente diminui a qualidade de vida do paciente resultando em readmissões hospitalares, onerando assim o sistema de saúde. Considera-se que o emprego de cuidados ubíquos, usando sensores e wearables, pode melhorar este processo reduzindo o número de readmissões hospitalares. Nesse âmbito, esse trabalho propõe o modelo UbHeart que emprega ciência da situação para identificar possíveis problemas cardíacos. Como contribuição científica o modelo prove o monitoramento da evolução da degradação dos sinais vitais do coração do paciente, através da detecção de possíveis situações de complicação cardíaca. Foram utilizados parâmetros de saúde estabelecidos pela sociedade brasileira de cardiologia. A avaliação do UbHeart foi realizada utilizando dois cenários de uso e apresentou resultados satisfatórios durante a análise.

\section{Introdução}

A readmissão em sistemas hospitalares tem crescido significativamente nos últimos anos. Apesar dos avanços nos cuidados de pacientes com insuficiência cardíaca (IC), os resultados após a internação não estão melhorando [Chaudhry et al. 2013]. Essa patologia é considerada um dos principais desafios médicos do nosso tempo [Ukena et al. 2012]. Sem acompanhamento médico diário os sinais fisiológicos dos pacientes são 
comprometidos e a internação torna-se inevitável. Este cenário recorrente diminui a qualidade de vida do paciente e resulta em retorno ao hospital, onerando assim o sistema de saúde.

Fatores associados ao aumento das taxas de readmissão incluem o descumprimento da medicação, à falta da procura de tratamento médico imediato quando os sintomas são exacerbados e automedicação [Anker, 2011]. Uma estratégia promissora é o telemonitoramento, que consiste em observar os sinais fisiológicos do paciente a fim de que os profissionais da saúde possam intervir mais cedo se houver evidência de deterioração clínica [Chaudhry et al. 2013].

A evolução da computação móvel e ubíqua tem favorecido o desenvolvimento de soluções que utilizam sensores e aplicativos móveis em benefício do ser humano. A ciência de situação permite correlacionar contextos logicamente agregados para definir o envolvimento de um usuário com determinado cenário mais complexo [Anagnostopoulos et al. 2007]. Particularmente, os smartphones tem capacidade significativa de detecção de contexto e interação com os usuários, as quais podem ser exploradas para construir poderosas aplicações na área da saúde [Scott et al. 2015]. Nesse âmbito, a área de cuidados ubíquos (do inglês Ubiquitous Care) permite o uso da computação móvel e ubíqua para o monitoramento da saúde do paciente em qualquer lugar e a qualquer momento, sem a necessidade da pessoa estar em uma clínica ou hospital. O propósito de se manter cuidados médicos de forma ubíqua, que vem sendo chamado de ubiquitous healthcare, é fornecer um serviço conveniente aos pacientes, facilitando o diagnóstico das condições clínicas [Gelogo et al. 2013].

No âmbito da saúde ubíqua, este trabalho está particularmente focado na insuficiência cardíaca e propõe o modelo denominado UbHeart. A Figura 1 apresenta o cenário em que o modelo proposto se insere. Primeiro é feito um diagnóstico prévio utilizando as informações adquiridas pelos sensores. Quando uma situação de risco é identificada um alerta é enviado ao paciente, podendo ainda ser comunicada aos responsáveis pelo paciente e ao hospital que aciona o médico.

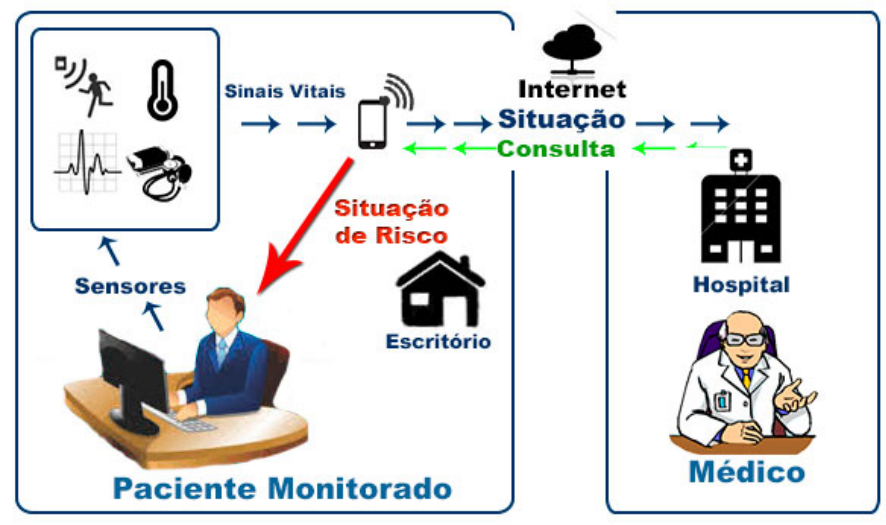

Figura 1. Cenário de monitoramento

A principal contribuição científica do trabalho é a possibilidade de reconhecer informações que possam identificar possíveis degradações fisiológicas e combiná-las a fim de caracterizar uma situação de risco cardíaco ao paciente, representando um auxílio nos cuidados médicos a fim de prevenir a readmissão hospitalar. Até o presente momento, não foram encontrados trabalhos que utilizam a ciência da situação aplicada ao monitoramento de sinais vitais de pacientes com insuficiência cardíaca. Durante as pesquisas, o estudo destacou como importante o monitoramento do progresso do 
paciente ao longo do tempo e a necessidade de analisar a ordem de classificação das medições a fim de auxiliar o médico quanto ao gráu de investigação [Kashem et al. 2008; Mulvaney et al. 2012]. Alguns trabalhos relacionados apontaram que as medições realizadas pelos sensores foram associados a perguntas efetuadas pelo médico ao paciente e também a códigos de cores informados no sistema [Kashem et al. 2008].

Este trabalho esta dividido em seis seções. A seção 2 descreve o modelo UbHeart. Uma avaliação baseada em um estudo de caso é apresentado na seção 3. A seção 4 apresenta trabalhos relacionados. Por fim, a seção 5 apresenta as conclusões.

\section{Modelo UbHeart}

O modelo proposto permite o monitoramento de sinais vitais utilizando equipamentos médicos interligados a uma rede Bluetooth a qual permite a aquisição dos sinais fisiológicos de forma mais cômoda ao paciente em ambiente residencial, como pode ser visto na Figura 2. O perfil do paciente é informado ao sistema pelo smartphone do paciente que, através dos sensores, adquire os sinais fisiológicos e monta um histórico de saúde, o qual é enviado ao hospital através da internet. A base de informações enviadas pelo smartphone a um serviço na nuvem que permite ao sistema analisar a situação de saúde baseada na evolução da degradação dos sinais vitais. Também e possível se basear em uma classificação de risco realizada, relacionando o perfil do paciente com os contextos adquiridos. O histórico de saúde enviado ao hospital permite que os médicos responsáveis pelo paciente saibam como está a sua saúde em um curto espaço de tempo, garantindo uma assistência rápida e assim prevenindo reinternações.

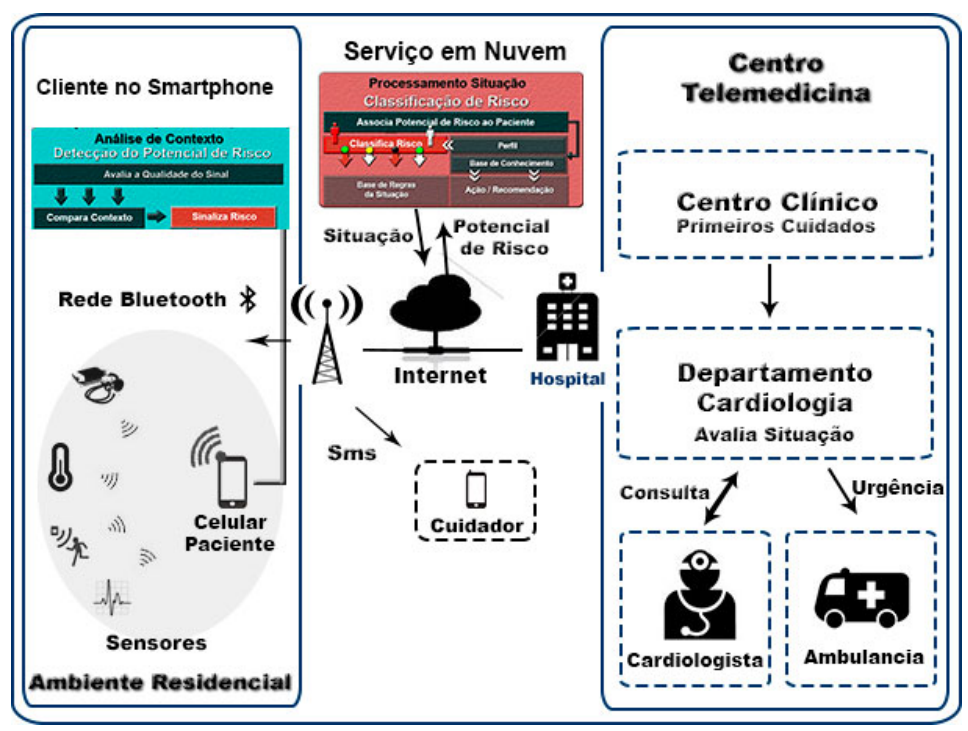

Figura 2. Visão Geral da Solução Proposta

O centro clínico realiza os primeiros cuidados passando informações ao paciente através de recomendação realizada pelo sistema, caso o estado de saúde necessite de assistência especializada é agendado uma consulta com o cardiologista, assim como, caso seja urgente, uma ambulância pode ser encaminhada ao local. Alterações de saúde são informadas ao cuidador por meio de mensagem pelo celular. $O$ paciente recebe várias recomendações de como proceder para melhorar seus índices de saúde ou como agir para evitar uma piora de seu estado de saúde mediante uma necessidade imediata. Podemos observar que o modelo propõe diversos recursos a fim de não permitir que o 
paciente fique sem cuidados médicos e que seus sinais fisiológicos não cheguem a níveis degradantes, como pode ser visto na Figura 3.

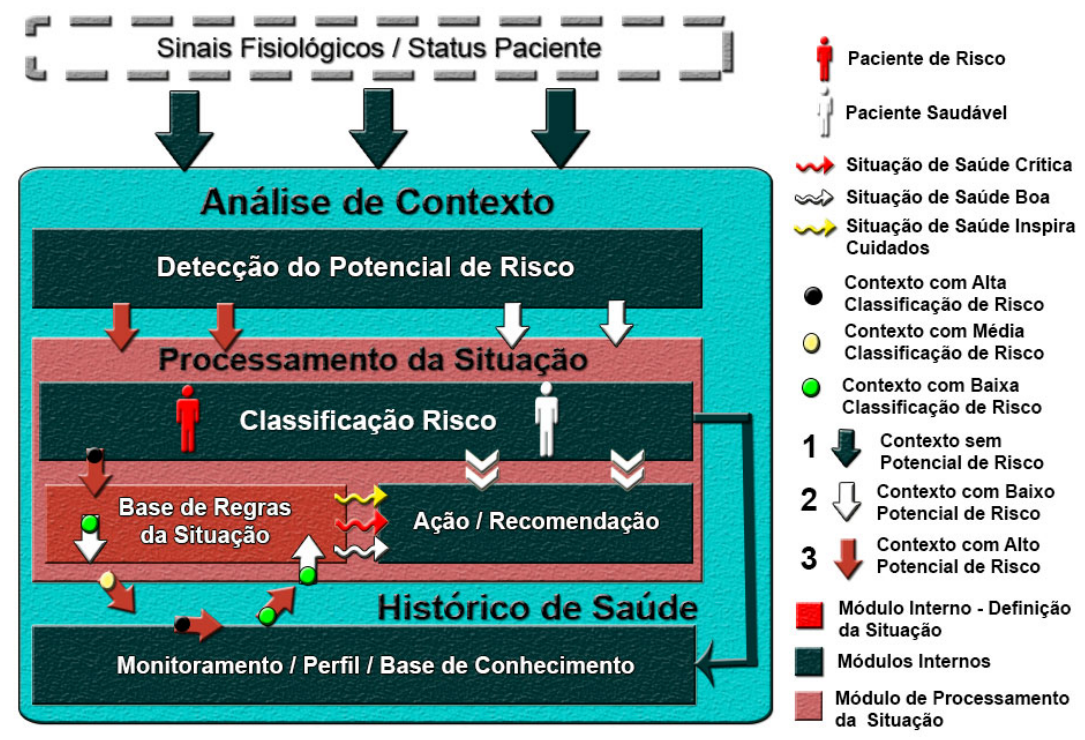

Figura 3. Modelo UbHeart

O modelo do serviço executando no smartphone do paciente é apresentada na Figura 4. Os sinais fisiológicos e o status do paciente são adquiridos pelos sistema (seta 1 na Figura). O componente Processamento de Situação reúne módulos responsáveis pela classificação do risco, regras e ações do sistema definição da situação de saúde e são processados por um serviço em uma nuvem computacional. Este serviço recebe os contextos a partir de uma análise realizada pelo módulo de detecção de potencial de risco no smartphone do paciente. O módulo que executa no smartphone do paciente é detalhado na Figura 4. O serviço é responsável pela detecção do potencial de risco do paciente. Nele o modelo sinaliza o potencial de risco com base nas informações de contexto e nas normas da Sociedade Brasileira de Cardiologia ${ }^{1}$.

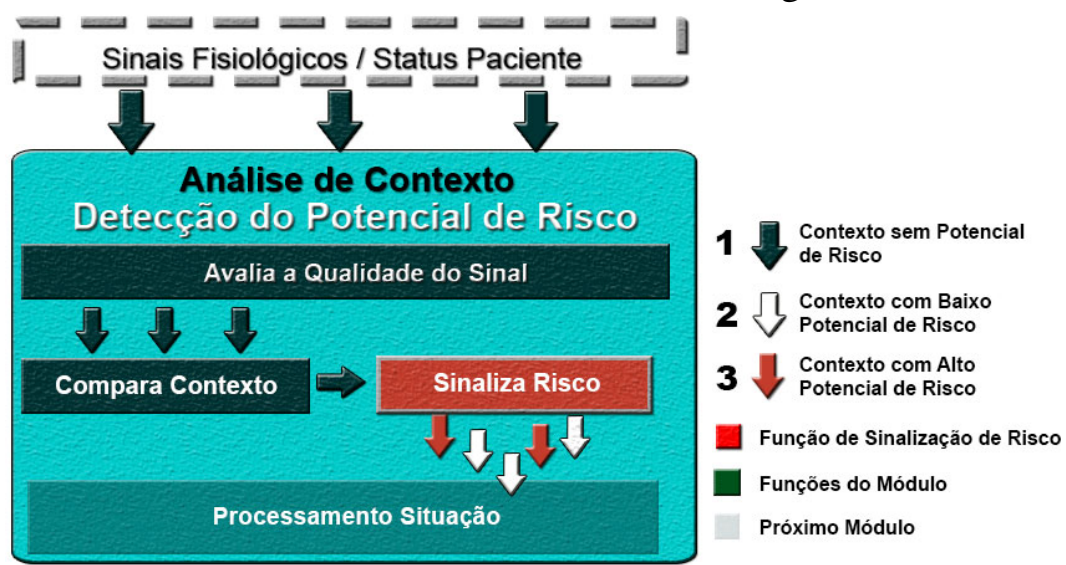

Figura 4. Serviço de Detecção de Potenciais Riscos do UbHeart no smartphone

Os contextos são adquiridos com base nos sinais fisiológicos do paciente (seta 1). A função avalia a qualidade do sinal verifica se a detecção do sinal fisiológico foi realizada com sucesso ou se apresenta alguma anormalidade, caso tenha ele é desprezado. Uma vez realizada esta etapa, o contexto é enviado a função compara

\footnotetext{
${ }^{1}$ http://publicacoes.cardiol.br/2014/diretrizes.asp
} 
contexto que é responsável por comparar os valores adquiridos com os estabelecidos pela sociedade brasileira de cardiologia em questões relacionadas a idade e patologia.

Cada contexto é comparado individualmente de acordo com o perfil do paciente no tempo em que ele é adquirido, e em seguida a função sinaliza risco é ativada. Caso o valor do contexto esteja fora do estabelecido pelos padrões, é sinalizado um potencial de risco de com cores (setas 2 e 3). Cabe ressaltar que a identificação do potencial não determina o risco, mas é extremamente importante para determinar sua classificação e posteriormente a situação, além de contribuir para modelar o conhecimento. Este módulo se baseia na análise de contexto e, portanto, não correlaciona os contexto, e sim os identifica individualmente passando para o serviço na nuvem do UbHeart.

O serviço na nuvem classifica o risco do paciente e é apresentado na Figura 5. De acordo com a classificação de potencial prévia e os contextos de risco identificado, a função associa potencial de risco ao paciente realiza a consulta a uma base de conhecimento. Existe duas análises possíveis, são elas: (i) comparar os contextos com sinalização de potencial de risco e o perfil do paciente com a base de conhecimento; caso não seja encontrado o perfil do paciente, (ii)o modelo aciona a função classifica risco que é responsável por classificar o risco associando com as diretrizes da sociedade brasileira de cardiologia.

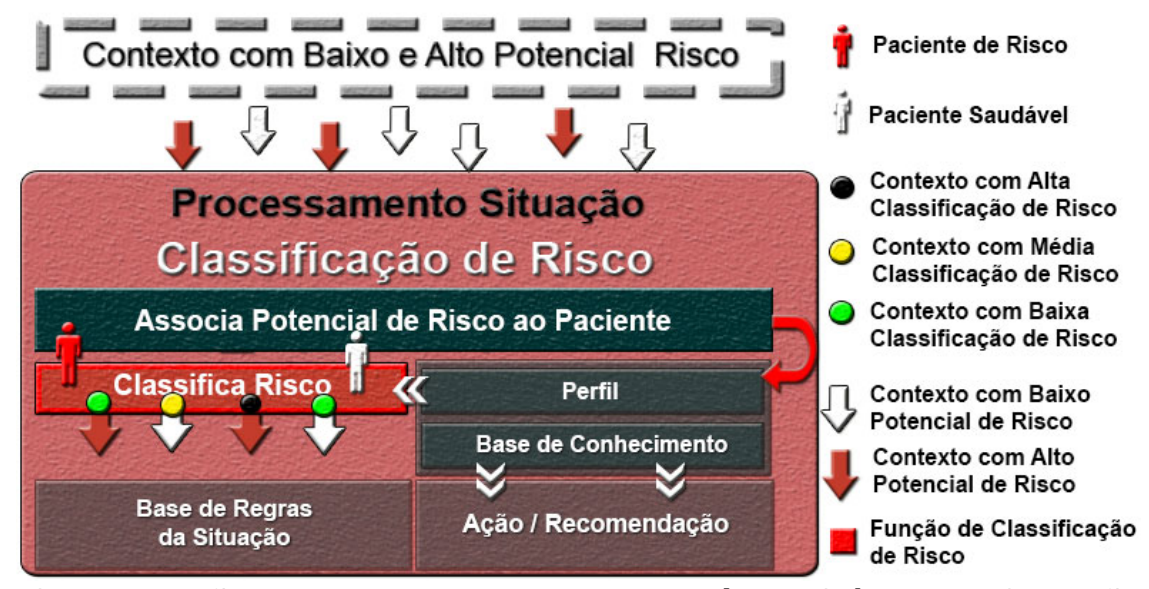

Figura 5. Classificação de Riscos do UbHeart através da Ciência de Situação

A classificação do risco é realizada em três níveis: preto, amarelo e verde, representando respectivamente alto, médio e baixo risco. Em seguida envia os contextos para o módulo base de regras da situação. $\mathrm{O}$ módulo realiza ações baseadas em diversas associações de contextos. As informações dos sinais fisiológicos identificados com potenciais de risco são associados aos contextos do perfil do paciente e a partir dos cálculos realizados, e dos padrões determinados pela Sociedade Brasileira de Cardiologia, classifica o risco que o paciente pode estar envolvido.

$\mathrm{O}$ funcionamento interno da base de regras da situação para o processamento da situação é apresentado na Figura 6. As regras são definidas de acordo com as funções determinadas, são elas: (i) Degradação - responsável por executar as atividades selecionar o perfil do paciente e verificar o nível de classificação do sinal fisiológico; (ii) Monitoramento - Iniciado por um evento enviado pela regra de degradação, é responsável por monitorar a evolução da degradação do sinal vital do paciente, além de monitorar os contextos relacionados, sejam advindos dos sensores ou de acordo com o perfil do paciente; (iii) Determinação da situação - esta regra determina em qual situação se encontra o paciente, correlacionado os valores adquiridos pela regra 
monitorar evolução da gradação com base nas diretrizes de cuidados do coração da Sociedade Brasileira de Cardiologia, em um intervalo de tempo, aos contextos de localização e perfil. Os resultados podem convergir para uma situação que, embora seja identificada com potencial e classificação de risco baixo, podem representar situações de risco iminente dependendo da localização e do contexto do perfil do paciente e viceversa. As regras da ação são acionadas de acordo com a classificação da situação. Que podem ser: (i) Nenhuma ação, caso não tenha histórico para a regra ou mesmo a situação não seja de risco; (ii) Recomendar, situação com classificações de risco baixa, aciona a regra que emite informações de orientações para o smartfone do paciente; (iii) Acionar cuidador, situações com classificações médias e altas as quais são reportadas ao cuidador do paciente e por fim ao centro de telemedicina.

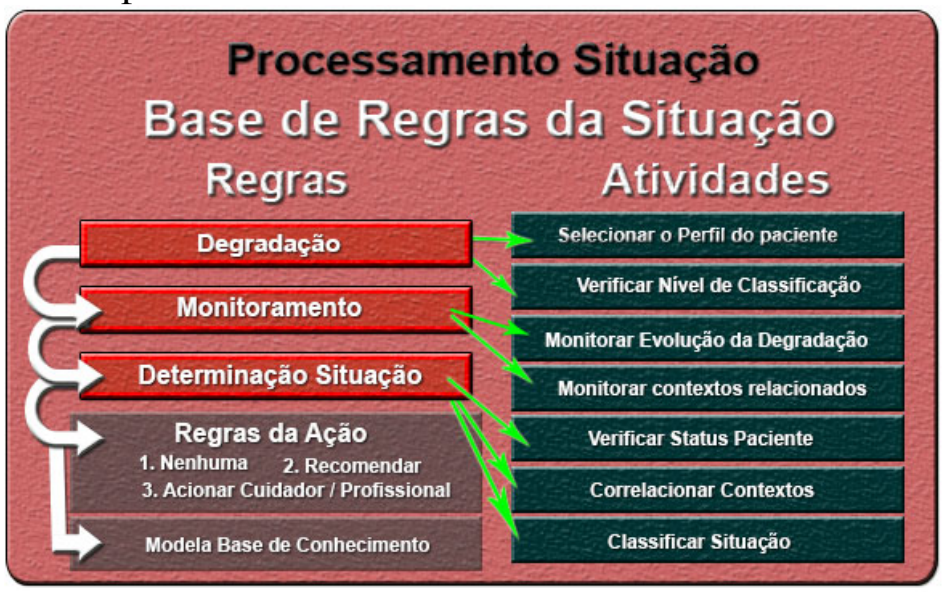

Figura 6. Módulo de Base de Regras e Ação

\section{Avaliação}

A comunidade científica tem empregado cenários para avaliação de aplicações ubíquas e sistemas sensíveis ao contexto [Satyanarayanan 2011; Souza 2014]. Partindo dessa estratégia, definiu-se um cenário para avaliações do modelo apresentado. O cenário demonstra a reação do UbHeart durante a coleta das informações adquiridas por sensores em um monitoramento de sinais vitais de um paciente com complicações cardíacas. O cenário usado foi:

"Os irmãos Lara e Mario resolvem levar sua mãe Andira, de 70 anos, ao shopping center perto de sua casa e por este motivo resolvem ir à pé. Mário e sua mãe são hipertensos e resolveram recentemente usar o sistema UbHeart para monitoramento de sua saúde. Na ocasião Lara foi cadastrada como cuidadora de ambos. Depois de alguns minutos passeando, Lara recebe uma ligação que informa do compromisso o qual havia esquecido e ela apressadamente resolve regressar com sua mãe e irmão para casa. No retorno, Lara recebe uma notificação do UbHeart em seu smartphone indicando alerta nos sinais vitais de Mario e Andira. Lara ao receber a mensagem resolve baixar o ritmo e perder o compromisso, em virtude da saúde de sua mãe e irmão. Logo em seguida recebe novamente outra notificação informando que a situação de saúde para ambos já melhorou e também uma orientação para encaminhar ambos para consulta médica."

Para avaliação do cenário, foi definido um protótipo baseado em Android que interage com um serviço em nuvem desenvolvido em Java. Os módulos de monitoramento de sinais vitais e de processamento de situação utilizados recebem os sinais vitais por uma rede Bluetooth. Foram desenvolvidas duas funcionalidades com o 
objetivo de assistir o paciente em questões de baixo e médio risco de saúde: a primeira direcionada para adquirir informações do perfil do paciente e a segunda direcionada a envio de mensagens a partir das regras de situação.

A prototipação do modelo UbHeart é constituída de dois serviços que interoperam: uma aplicação móvel e outra que processa em nuvem. A Figura 7 apresenta as telas da aplicação cliente. A Figura 7(a) apresenta as opções principais da aplicação, que são: definir o perfil, chamar o cuidador, inserir o peso (dado importante para a identificação de risco que é monitorado manualmente) e status, para obter o atual nível de risco. A Figura 7(b) apresenta a tela de cadastro do perfil do paciente. Nela é possível inserir informações que definem o perfil do paciente tais como: altura, idade, status de saúde e sexo que são necessárias para detecção do potencial de risco.

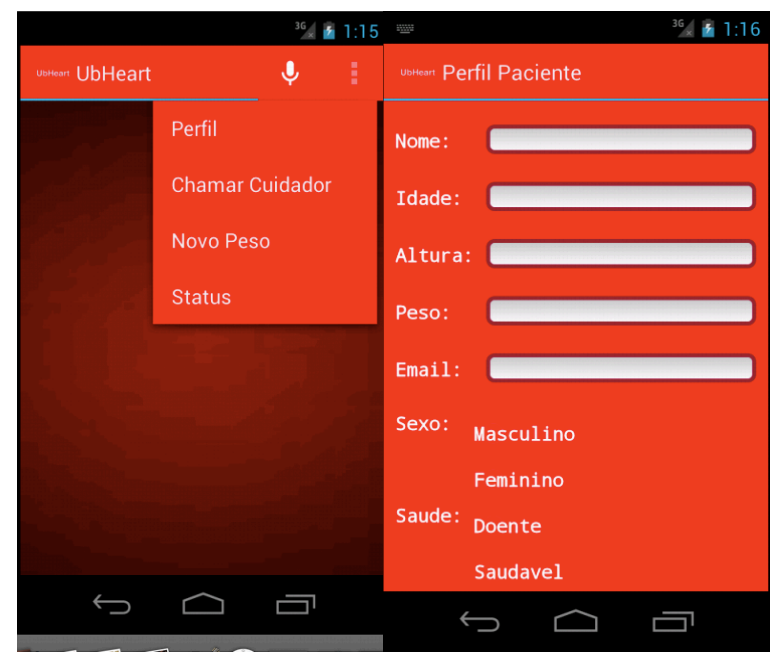

(a)

(b)

Figura 7. Protótipo do UbiHeart desenvolvido para Android

Para utilizar o UbHeart é necessário cadastrar algumas informações as quais fazem parte do perfil do paciente. Andira e Mário informaram o seu peso, idade, patologia e as informações complementares de seu perfil. Durante o passeio, foram monitorados sinais de pressão arterial (PA) diastólica e sistólica e batimento cardíaco, mas nenhuma ocorrência foi sinalizada, uma vez que tanto Mário como Andira haviam tomado o remédio para hipertensão. O mesmo não ocorre quando Lara resolve apressadamente retornar. Sem perceber durante o retorno a pressão arterial de Andira sobe rapidamente. O módulo de potencial de risco do sistema no smartphone do paciente recebe as informações de PA e batimento cardíaco através dos sensores e realiza algumas ações, são elas: 1. Classifica o potencial de risco da PA e batimento cardíaco como alto, em função da idade de Andira. Todavia, classifica a PA e batimento cardíaco de Mário como baixo, uma vez que ele é jovem.

É enviado para Andira um janela questionando se ela esta realizando alguma atividade física e para Mário nenhum questionamento é feito, uma vez que seu potencial de risco é baixo. O sistema no smartphone envia as informações já com as classificações de potencial de risco via rede $3 \mathrm{G}$ para o módulo de processamento de situação em nuvem, que compara com a base de conhecimento montada através das diversas situações ocorridas em ambos.

Nenhuma ocorrência foi encontrada, pois eles estão sendo monitorado pelo sistema a pouco tempo. Sendo assim o sistema classifica o risco de acordo com o perfil 
do paciente, para Andira são correlacionados os contextos de peso, idade, PA, patologia, batimento cardíaco e questionamento realizado. Sua classificação de risco é definida como alta, uma vez que sua pressão arterial média foi calculada e esta alta, sua idade é avançada e o seu batimento cardíaco aumentou e ela não estava fazendo exercício físico. Para Mário sua classificação foi definida como média, uma vez que foi correlacionado sua pressão arterial média, peso, batimento cardíaco e patologia e somente sua pressão arterial e peso estavam pouco alterados. O UbHeart, através do módulo de base de regra de situação passa a monitorar os sinais vitais de ambos a fim de identificar possível evolução na degradação dos sinais fisiológicos de Mário e Andira.

Depois de alguns segundos, o sistema verificou que a pressão de Andira e Mário aumentou ainda mais e seus batimentos cardíacos também. Em seguida o sistema define a situação de risco alto para ambos, aciona a base de regras de ação que envia notificação para Lara comunicando o fato e insere na base de conhecimento a classificação como situação de risco alta para as variações de PA e batimento cardíaco. A continuidade do monitoramento, em função da redução de ritmo de ambos, classifica o risco como baixo e permite uma tranquilidade maior para ambos.

\section{Trabalhos Relacionados}

Foram consideradas propostas relacionadas com o tema deste trabalho, particularmente modelos de sistemas de monitoramentos de sinais vitais que utilizam características da computação ubíqua e ciência de contexto. O trabalho [Mulvaney et al. 2012] descreve um modelo de utilização de uma rede de sensores sem fios para identificar medições e fornecer indicadores para análise de tendências e envio de possíveis alerta de complicações ao paciente. As medidas de glicose, pressão arterial, temperatura e respiração foram adquiridas por sensores e enviadas através de um dispositivo móvel à clínica. Uma camada comunicação provê o repasse das informações ao médico remoto e a clínica. $\mathrm{O}$ estudo aponta a importância para análise de tendência para monitorar o progresso do paciente ao longo do tempo e assim determinar a situação do paciente e pode dar alertas antecipados de possíveis complicações que poderiam se desenvolver.

[Kashem et al. 2008] propõe um modelo aplicado a telemedicina que utiliza informações de peso, pressão e batimentos cardíacos, para encontrar possíveis situações de risco a pacientes. O processamento é realizado em um servidor e o estado clínico do paciente é definido utilizando medições que os pacientes informam manualmente. $\mathrm{O}$ sistema proposto está dividido em três partes: pacientes, provedores de domínios e servidor e permite aos pacientes enviar dados diretamente ao banco de dados e receber os diagnósticos fornecidos por um médico. Embora o trabalho destaque a relevância da situação de saúde do paciente, o modelo descreve a qualificação da situação através da análise das medições relacionadas a perguntas feitas ao paciente durante o telefonema realizado pela enfermeira.

O modelo proposto por [Winkler et al. 2011] permite adquirir sinais fisiológicos de pacientes através de equipamentos médicos portáteis de eletrocardiograma, pressão arterial, peso corporal e medidas de auto-avaliação que são processadas em um servidor remoto. As medidas são classificadas individualmente com a utilização de código de cores e recebidas gerando eventos de acordo com as regras de priorização médico para iniciar um processo de revisão guiado por fluxo de trabalho no sistema e uma avaliação mais aprofundada por parte dos profissionais médicos. $\mathrm{O}$ modelo permite a alteração da ordem de visualização dos dados processados no centro medico de acordo com seu nível 
crítico e ainda a interação do paciente o qual realiza sua auto-avaliação utilizando uma escala de um código de cores. Medições recebidas geram eventos de acordo com as regras de priorização médico para iniciar um processo de revisão guiado por fluxo de trabalho e uma avaliação mais aprofundada por parte dos profissionais médicos.

[Suh et al. 2011] definem um modelo para realizar o monitoramento de sinais vitais de pacientes com IC. As medidas de peso, atividade física, pressão arterial e frequência cardíaca são adquiridas por sensores. A arquitetura foi construída em três camadas: (1) recebe as medidas relacionadas com a saúde dos pacientes e as transmite; (2) recebe os dados para análise de regressão linear, verifica a ausência de alguma delas e a sua integridade; (3) armazena os dados para consultas posteriores.

Por fim, foi analisado o modelo de [Costa et al. 2012] que utiliza um correio eletrônico e os seus protocolos para apoiar o núcleo do sistema de informação de telemedicina. São empregados dois métodos: o primeiro em tempo real, em que os participantes podem enviar e receber informações quase que instantaneamente; o segundo para armazenamento e encaminhamento em uma localização física. O modelo proposto utiliza o e-mail para carregar exames ao hospital e retornar relatórios.

A Tabela 1 apresenta um resumo das características dos trabalhos relacionados. O monitoramento de sinais vitais para pacientes com insuficiência cardíaca com uso de sensores e a associação das medições a perguntas, ou ainda, analisando o conjunto de informações para definir o risco do paciente, foram características encontradas em todos os trabalhos relacionados e qualifica o uso manual da ciência de situação. Na maioria dos trabalhos foram utilizados dispositivos móveis e equipamentos médicos idênticos para aquisição dos sinais vitais. Além disso, contextos foram utilizados para identificar/sinalizar possíveis riscos. Algumas características ubíquas foram detectadas, todavia nenhum aplicabilidade automática da ciência da situação foi encontrada.

Tabela 1. Comparação de características de trabalhos relacionados

\begin{tabular}{|c|c|c|c|c|c|}
\hline Característica & $\begin{array}{l}\text { [Mulvaney et al. } \\
\text { 2012] }\end{array}$ & $\begin{array}{c}\text { [Kashem et al. } \\
\text { 2008] }\end{array}$ & [Winkler et al. 2011] & [Suh et al. 2011] & [Costa et al. 2012] \\
\hline $\begin{array}{l}\text { Uso de } \\
\text { dispositivos } \\
\text { médicos }\end{array}$ & $\begin{array}{l}\text { Medidor de } \\
\text { glicose, } \\
\text { esfigmomanô- } \\
\text { metro } \\
\end{array}$ & Esfigmomanômetro & $\begin{array}{l}\text { Esfigmomanômetro, } \\
\text { sensores e balança }\end{array}$ & $\begin{array}{c}\text { Esfigmomanômetro, } \\
\text { monitor atividade, balança }\end{array}$ & ECG \\
\hline $\begin{array}{c}\text { Uso de } \\
\text { dispositivos } \\
\text { móvel }\end{array}$ & PDA, celular & Não & PDA, celular & Smartphone & Não \\
\hline $\begin{array}{l}\text { Contextos } \\
\text { Utilizados }\end{array}$ & \begin{tabular}{|c|} 
Glicose, pressão \\
arterial, \\
temperatura, \\
respiração \\
\end{tabular} & $\begin{array}{l}\text { Peso, pressão } \\
\text { arterial e } \\
\text { frequência } \\
\text { cardíaca } \\
\end{array}$ & $\begin{array}{c}\text { Peso, pressão, } \\
\text { eletrocardiograma e } \\
\text { oxigênio }\end{array}$ & $\begin{array}{c}\text { Peso, pressão arterial, } \\
\text { frequência cardíaca e } \\
\text { atividade }\end{array}$ & $\begin{array}{c}\text { Eletrocardiograma, } \\
\text { ecocardiografia }\end{array}$ \\
\hline $\begin{array}{l}\text { Ciência de } \\
\text { Contexto }\end{array}$ & $\begin{array}{c}\text { Códigos de cores } \\
\text { atribuídas as } \\
\text { medições }\end{array}$ & $\begin{array}{l}\text { Gráficos no } \\
\text { hospital }\end{array}$ & $\begin{array}{l}\text { Códigos de cores } \\
\text { atribuídas as } \\
\text { medições }\end{array}$ & $\begin{array}{c}\text { Escalas de valores e cores } \\
\text { atribuídas as medições }\end{array}$ & $\begin{array}{l}\text { Curva do ECG } \\
\text { sinaliza estado }\end{array}$ \\
\hline $\begin{array}{c}\text { Identificação / } \\
\text { Sinalização de } \\
\text { possíveis } \\
\text { Risco }\end{array}$ & \begin{tabular}{|c|} 
Medição com \\
Código de cor \\
vermelha sinaliza \\
risco e aciona \\
profissionais \\
\end{tabular} & $\begin{array}{c}\text { Gráfico negativo } \\
\text { aciona profissional }\end{array}$ & $\begin{array}{l}\text { Medição com } \\
\text { Código de cor } \\
\text { vermelha sinaliza } \\
\text { risco e aciona } \\
\text { profissionais }\end{array}$ & $\begin{array}{c}\text { Medição com Código de } \\
\text { cor vermelha sinaliza risco } \\
\text { e aciona profissionais. }\end{array}$ & $\begin{array}{l}\text { Curva do ECG } \\
\text { sinaliza estado } \\
\text { degradado }\end{array}$ \\
\hline $\begin{array}{c}\text { Características } \\
\text { Ubíquas }\end{array}$ & $\begin{array}{l}\text { Ações realizadas } \\
\text { a partir da } \\
\text { sinalização do } \\
\text { risco }\end{array}$ & $\begin{array}{l}\text { Associação dos } \\
\text { contextos a } \\
\text { perguntas } \\
\text { realizada pela } \\
\text { enfermeira }\end{array}$ & $\begin{array}{c}\text { Ações realizadas a } \\
\text { partir da sinalização } \\
\text { do risco. }\end{array}$ & $\begin{array}{c}\text { Profissionais acionados a } \\
\text { partir da sinalização de } \\
\text { risco. }\end{array}$ & $\begin{array}{c}\text { ECG com sinal } \\
\text { degradado altera } \\
\text { mensagem de } \\
\text { email }\end{array}$ \\
\hline $\begin{array}{c}\text { Uso de ciência } \\
\text { de situação da } \\
\text { saúde }\end{array}$ & Manual & Manual & Manual & Manual & Manual \\
\hline
\end{tabular}




\section{Conclusão}

O Modelo UbHeart permite o monitoramento de sinais vitais do coração e utiliza como base a ciência da situação. O protótipo desenvolvido se mostrou uma ferramenta eficaz na identificação de situações de riscos cardíacos em que pacientes podem estar envolvidos. A evolução da degradação dos sinais vitais e a correlação entre os contextos adquiridos com os perfis dos pacientes foram fatores determinantes para a definição do nível de risco do paciente. Ações de recomendação enviadas pelo sistemas permitem que o tratamento seja rápido, através de uma intervenção médica.

Para trabalhos futuros pretende-se realizar definições de situação admitindo a ausência de contextos utilizados durante a análise da evolução da degradação. Além disso, será realizado um estudo de caso com pacientes em um ambiente controlado para identificar o grau de influência do ambiente em que o paciente se encontra na determinação da situação em que ele esta envolvido.

\section{Agradecimentos}

Os autores gostariam de agradecer ao CNPq pelo apoio a esta pesquisa.

\section{Referências}

Anker, S. D., Koehler, F., and Abraham, W. T. (2011). Telemedicine and remote management of patients with heart failure. The Lancet, 378(9792), 731-739.

Chaudhry, S. I et al. (2010). Telemonitoring in patients with heart failure.New England Journal of Medicine, 363(24), 2301-2309.

Costa, C., and Oliveira, J. L. (2012). Telecardiology through ubiquitous Internet services. International journal of medical informatics, 81(9), 612-621.

Gelogo, Y. E., and Kim, H. K. (2013). Unified Ubiquitous Healthcare System Architecture with Collaborative Model. International Journal of Multimedia and Ubiquitous Engineering, 8(3).

Kashem, A., Droogan, M. T., Santamore, W. P., Wald, J. W., and Bove, A. A. (2008). Managing heart failure care using an internet-based telemedicine system. Journal of Cardiac Failure, 14(2), 121-126.

Mulvaney, D., Woodward, B., Datta, S., Harvey, P., Vyas, A., Thakker, B., and Istepanian, R. (2012). Monitoring heart disease and diabetes with mobile internet communications. International journal of telemedicine and applications, 2012, 12.

Satyanarayanan, M. (2011). Mobile computing: the next decade. ACM SIGMOBILE Mobile Computing and Communications Review, 15(2), 2-10.

Scott, R. E., Mars, M., Scott, R. E., and Mars, M. (2015). Telehealth in the developing world: current status and future prospects. OncoTargets and Therapy, 8, 289-294.

Souza A. et al. (2014). Uma Abordagem Ubíqua Consciente de Situação para Avaliacao de Metas Terapêuticas em Ambiente Hospitalar. SBCUP 2014.

Suh, M. K et al. (2011). A remote patient monitoring system for congestive heart failure. Journal of medical systems, 35(5), 1165-1179.

Ukena, C., and Böhm, M. (2013). Management of heart failure: are specialists really needed? European heart journal, 34(6), 416-418.

Winkler, $S$ et al. (2011). A new telemonitoring system intended for chronic heart failure patients using mobile telephone technology - feasibility study. International Journal of Cardiology, 153(1), 55-58. 